\title{
Comparison of Halogen and Xenon Headlamps in Terms of Pedestrians' Visibility
}

\begin{abstract}
This case study presents a comparison of visibility and recognisability (discernibility) of objects - pedestrians - on the road in rural areas at night on encounter with low beam headlights fitted with halogen lights and xenon (high-intensity discharge) lights in one and the same vehicle model. The study was inspired by the results of dynamic measuring of the reactions of vehicle drivers to the presence of pedestrians on the right side of the roadway, where the results indicated subsequent reactions of drivers driving a vehicle fitted with xenon discharge lights. The study is therefore concluded with a static comparison that confirms the factual findings.
\end{abstract}

Keywords: traffic accident, visibility, recognisability, lighting, xenon, halogen.

\section{Introduction}

The Institute of Forensic Engineering of the Brno University of Technology has been studying the behaviour of drivers in connection with traffic accidents for a considerable time. Recently, the research concentrated on drivers' behaviour at night on roads without artificial lighting. The impulse for the study stems from the unfavourable statistics of the causes and consequences of traffic accidents with pedestrians in the Czech Republic. Out of the pedestrians fatally injured in a traffic accident annually, over $60 \%$ were killed at might (comparison shows a significant difference between daytime and night time traffic density), thereof approximately $90 \%$ fatal accident took place on rural roads, i.e., roads without public lighting. The main reason is the poor visibility of pedestrians due to their non-contrasting clothing, vehicle speed, limitation of beam distance due to headlight design, hence limited options on the part of drivers to avoid an accident. Until 2016, pedestrians in the $\mathrm{CR}$ have no legal obligation to use reflective elements when moving on dark road at night, so that driver should expect even the presence of pedestrians in dark clothing, who blend with the background, and adjust the speed of their vehicle accordingly.

In practice, it means that drivers should adjust the speed so as to be able to recognize a pedestrian from a distance that allows them to stop their vehicle safely. The distance necessary for stopping the vehicle consists of the track covered by the vehicle during the driver's reaction interval, i.e., the time when the vehicle is not slowing down, and the actual braking distance which depends on the vehicle's braking capability. Generally, one may speak of a dependence of the vehicle's braking distance on the vehicle's speed at the time, see Fig. 1.

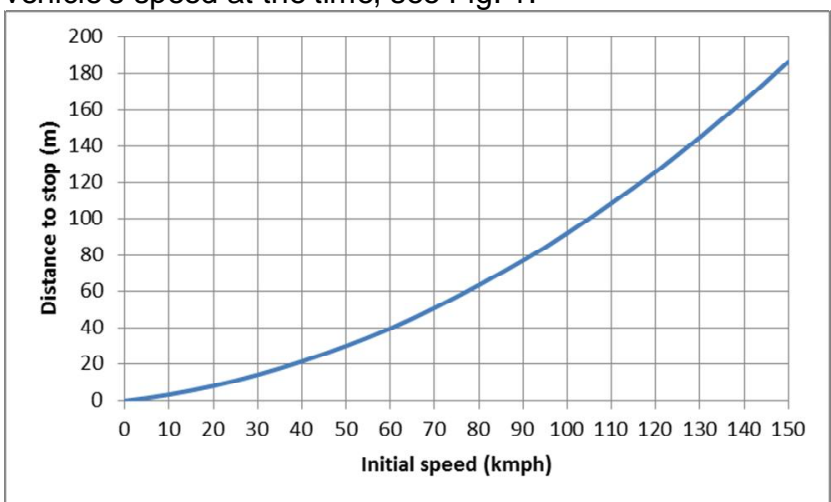

Fig.1. Dependence of braking distance on vehicle speed.
Generally, the distance at which it is possible to spot a pedestrian at low-beam lights is approx.. 30 to 60 meters, depending on his clothing. However, if he is wearing reflective clothing, the distance may be even more than 100 meters. Thus, a driver driving at night with low-beam lights should maintain a speed of up to $50 \mathrm{~km} / \mathrm{h}$ even on rural roads to be sure to be able to stop in time if he spots a pedestrian in dark clothing.

The aim of our survey (projects OP FMP CZ0069 2010 to 2011 and TACR TD020239 - 2014 to 2015) was to identify the real chance of drivers to spot a pedestrian/ figurant in real road traffic under various conditions (background, road class, figurant's color, type of headlight). Measuring was done with the aid of an eye tracker, complemented in the more recent project by data from the vehicle's control units and records from differential GPS.

Already records from the measured route in the first of the above projects indicated that drivers whose vehicles were fitted with xenon lights needed more time to focus on pedestrians than drivers in vehicles fitted with halogen lights. However, the project included several other scenarios (figurants in urban areas, figurants crossing the road, etc.), so that the volume of data collected on the reaction to pedestrians in rural areas did not have adequate reporting value.

Other projects therefore concentrated strictly on rural roads, whereby not only the number of figurants, but especially the number of drivers was increased. The results of these measurements will be presented herein below, as well. 


\section{Experimental measurements}

The measuring process

Measuring was divided into two parts - dynamic and static. Dynamic measuring involved driving along a certain route and monitoring the angle of his eyes using an eye tracker fitted with a recording device, and simultaneous collecting of data from the vehicle's control units (speed, rpm, acceleration pedal position, etc.) and from a differential GPS (position with accuracy to the centimeter, acceleration, speed, etc.). Static measuring was done by means of gradual approximation to the figurant up to a distance that corresponded to the spotting of the figurant during the dynamic test, with subsequent measuring of the brightness of the figurant as well as the background (see below). Taking into consideration the differences between the reaction of drivers driving a vehicle fitted with xenon lights and those with halogen lights, we took comparative measurements from the same distance with the same figurants using two identical vehicles that differed solely by the headlight design.

\section{The measured route}

The route we chose had to meet the following parameters:

- the route had to be circular to facilitate switching of drivers, calibration of technology, etc.;

- different road classes had to be represented evenly (Class I, II, and III);

- the route had to be adequately long to facilitate distribution of a suitable number of figurants;

- a minimal number of the route's sections had to be located in urban areas:

- the route had to be within a reasonable distance from the realization team.

These parameters were fulfilled best by the route Lednice-Valtice-Břeclav-Lednice - approx. $20 \mathrm{~km}$ long, containing about one third of each road class, whereby the Class I section (Valtice-Břeclav) is a divided road with a central dividing line and both guide lines; the Class II section (Břeclav-Lednice) and Class III section (LedniceValtice) only have a central dividing line. Horizontal traffic markings not only have impact on pedestrians' visibility as such, and the guiding side line may also form a contrasting part of the figurant's background, but it also facilitates the driver's orientation on the road, thus improving his attention capacity that he has to devote to his orientation. There were 12 figurants on the route, 4 in each of the sections.

For the purposes of static measuring, we selected a straight road without disturbing factors - this is where the illumination parameters of both vehicles were tested, from the same distance, on three figurants wearing different clothing (see below).

\section{The vehicles used}

For the purposes of measuring, we used two Škoda Yeti vehicles $\left(1^{\text {st }}\right.$ generation after facelift, made in 2012). The only substantial difference between them were the headlights: one of them was fitted with halogen lights, using $\mathrm{H} 7$ bulb, and the other with xenon lights (high-intensity discharge).

\section{The drivers}

For the measuring tests, we hired a total of 26 experienced drivers. The drivers were not acquainted with the objective of the measuring or the distribution of the figurants. Prior to the tests, all drivers underwent a check of their vision and sensitivity to contrasts.

The measuring technology
The following technology was used for the individual measurements:

Dynamic measurements:

- Eye tracker Viewpointsystem® - a camera that takes the images before the driver and a camera tracking the movements of the driver's right eye. Both cameras were fitted with a lens without an IRcut filter and optional additional illumination with an IR lamp.

- The vehicle's control units - using a wireless (BT) module in the vehicle's OBDIl compartment recorded data on real-time speed, rpm, and position of the acceleration pedal. The data were synchronized with records from the video camera that monitored the dashboard (use of blinkers, switching of low and high beam lights + supplementary infra-diode signalizing brake pedal activation).

- Differential GPS RT3002 from Oxford Technical Solutions, i.e., one GPS unit in the vehicle with additional sensors and a stable base approx. in the middle of the measured circuit, mutually interconnected with a radio connection - data saved synchronously with the former; synchronization with eye tracker done subsequently as part of evaluation.

Static measurements:

- Mechanical pedometer for distance measuring (wheel).

- LumiDISP - software for analyzing the luminance conditions based on evaluation of image data from digital photos. Application create high dynamic range image of luminance values and allows evaluation of contrast of selected regions. Calibrated digital camera NIKON D7000 was used for data acquisition.

\section{The figurants}

Twelve differently dressed figurants were distributed along the route, who simulated regular pedestrians walking along the right side of the roadway in the opposite direction. Two figurants were wearing white, two were wearing black, and the others were wearing various leisure-type of clothing. Selected figurants wore a safety vest or reflective tape on their sleeves.

During the static tests, in addition to dynamic measuring, the illumination parameters of the headlight of both vehicles were verified and compared using three figurants wearing white, black, and leisure-type of clothing.

\section{The mode of evaluation}

\section{Evaluation of dynamic tests}

The video records of dynamic tests, frame by frame, were analyzed using an SW environment developed by the Institute, whereby the moment at which the angle of the driver's eye looking in the figurant's direction changed. Simultaneously, we checked whether the change was subsequently confirmed by the driver's action (glance in the rear mirror, blinker activation, braking). The position data from the GPS unit were thereupon computed and, for double verification, also the distance from the figurant at individual moments of the driver's behavior.

\section{Evaluation of static measurements}

The static tests were used for identifying the contrast value of each figurant against his background when illuminated by the vehicle from a distance defined by 
dynamic tests. Furthermore, an evaluation of the contrast values was made by comparative measuring of both vehicles at the same distance $(20$ to $80 \mathrm{~m})$, with the same figurants (in white, black, and leisure clothing). For this particular purpose, brightness maps were used - see Fig. 2.

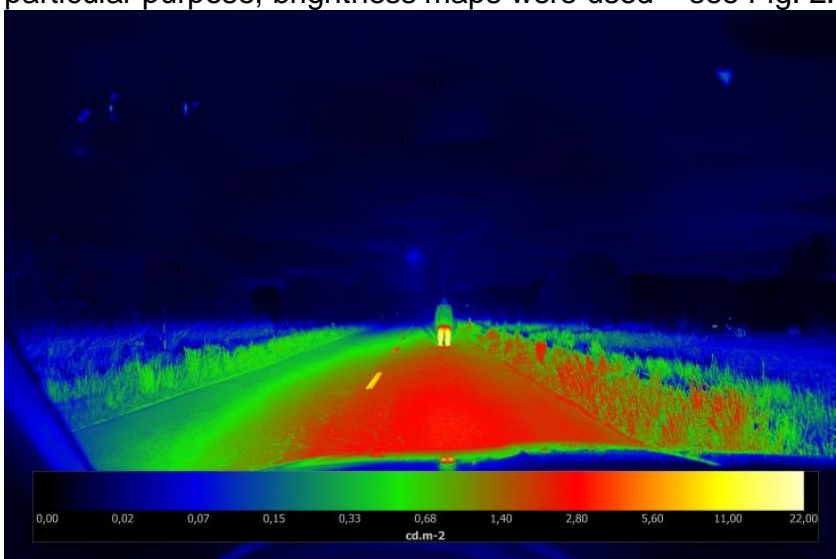

Fig.2. Example of a brightness map - white figurant $20 \mathrm{~m}$ before the vehicle with xenon lights.

Contrast $\mathrm{C}$ was defined on the basis of the brightness of the lower part of the figurant's legs (the first illuminated body part at low-beam lights) $\mathrm{L}_{\mathrm{obj}}$ and the brightness of his background (Fig. 3) L back using the following formula:

$$
C=\frac{L_{o b j}-L_{b a c k}}{L_{b a c k}}
$$

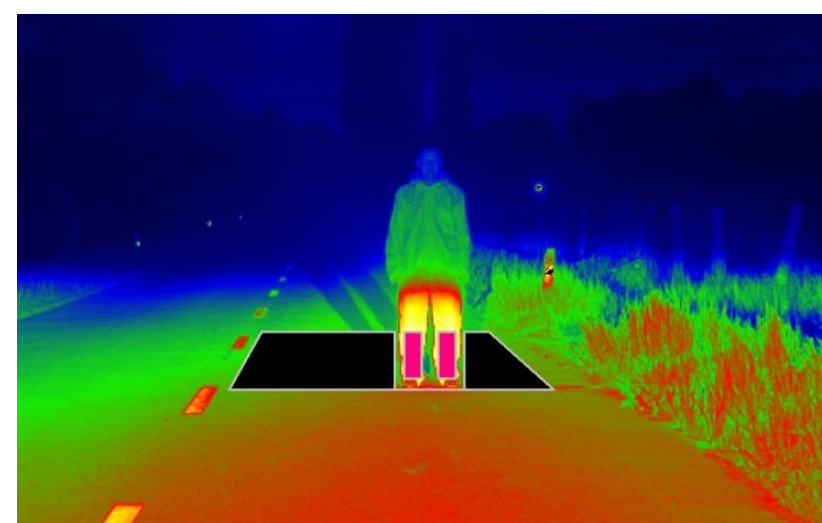

Fig.3. Areas evaluated for contrast - average brightness of purple areas $=$ object brightness, average brightness of black areas $=$ background brightness .

\section{The measurement results}

\section{Dynamic measurements}

Individual situations evaluated using records from dynamic tests were divided into categories by evaluation capability and subsequent credibility of the results. Most credible were considered to be situations where the driver distinctly changed the direction of his glance towards the figurant and his immediate action afterwards confirmed that he has registered the figurant and is about to by-passing him (blinker, deceleration, or braking). On the contrary, least credible results indicated minimal change of the view angle and the driver did not use any other means of vehicle control but the steering wheel. The last two categories contained measurements that could not be evaluated (the driver monitors the right side of the road all the time, so that it cannot be recognized when he registered the figurant), or a situation where his attention was influenced by other factors (driving behind another vehicle, blending of the driver's eyes by a vehicle from opposite direction, etc.). The resulting distances, at which the drivers registered the figurant in evaluable situations, are presented in the following diagram (Fig. 4).

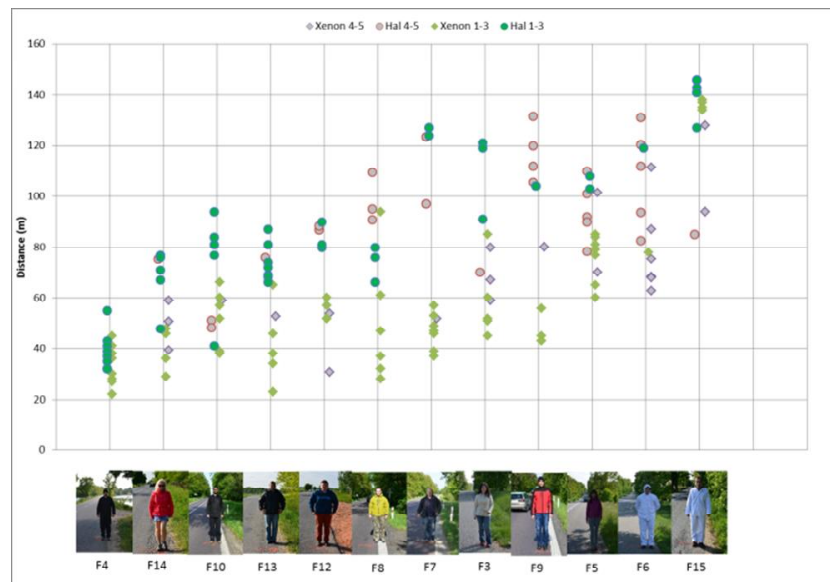

Fig.4. The distance of driver's first reaction to individual figurants.

The results marked Xenon 1-3 and Hal 1-3 may be considered the most credible. For clarity, we also added the photographs of the drivers during daytime. The diagram corroborates the aforementioned finding: namely, most of the drivers whose vehicle were fitted with xenon lights (rhombus shape) reacted to the figurants later than the drivers with halogen lights (circles). In practice, the shortening of the distance between the figurant and the vehicle at the moment of the driver's first optical reaction would substantially impact on the degree of the risk of a traffic accident as well as the technical and legal consequences potentially arising therefrom. For instance, the distance necessary for stopping a vehicle safely at a speed of $90 \mathrm{~km} / \mathrm{h}$, including the driver's reaction, is about 80 meters. Mere 5 out of 12 figurants (thereof 2 in white clothing) were registered by drivers with xenon lights at a sufficient distance, whereas the drivers of vehicles with halogen lights reacted in sufficient time to at least 10 out of 12 figurants.

\section{Static measurements}

The purpose of static measuring was to identify the illumination parameters in concrete cases, based on the evaluation of dynamic measurements. It should be noted that static measuring cannot take into consideration the vehicle's dynamic movements, especially its up and down rocking due to crossing over an uneven roadway, where this phenomenon may extend or shorten the distance illuminated by the vehicle momentarily.

The processing of these measurements is still in progress, yet we already have the availability of the results of comparative illumination parameters of both vehicles from the same distance using 3 identical figurants. As stated above, the results of dynamic tests indicate that drivers with xenon lights reacted later, i.e., at a shorter distance, compared to drivers with halogen lights. This comparison was supposed to confirm or disprove the hypothesis that xenon lights are less effective in terms of visibility of obstructions at night.

Individual tests also involved evaluation of brightness, with subsequent assessment of the contrast of the lower part of the figurants' legs (at low-beam lights, the figurant is illuminated by the approaching vehicle gradually from the bottom to the top, due to the headlight's angle) against the background at the same height.

The evaluation of all the measurements allowed us to identify the following relation between the contrast created 
by the figurant's illumination by the vehicle and the vehicle's distance from the figurant:

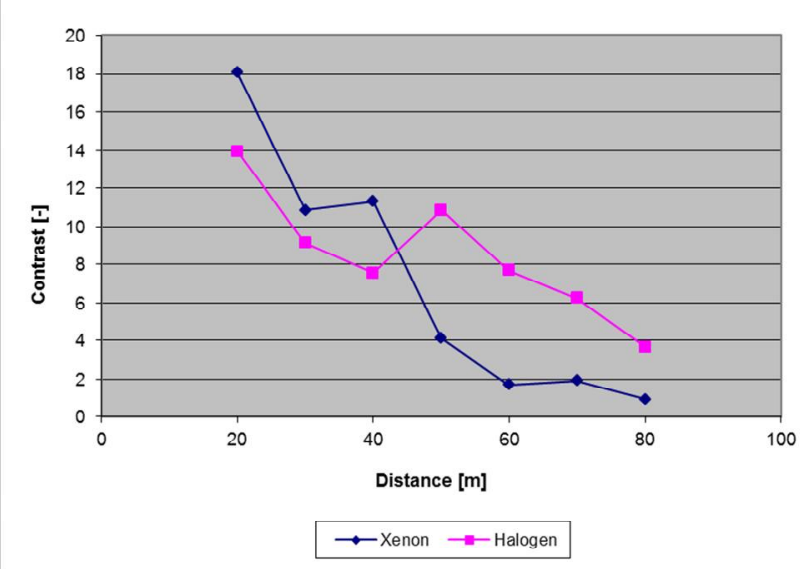

Fig.5. The effect of contrast of a figurant in white clothing at certain distances of the vehicle.

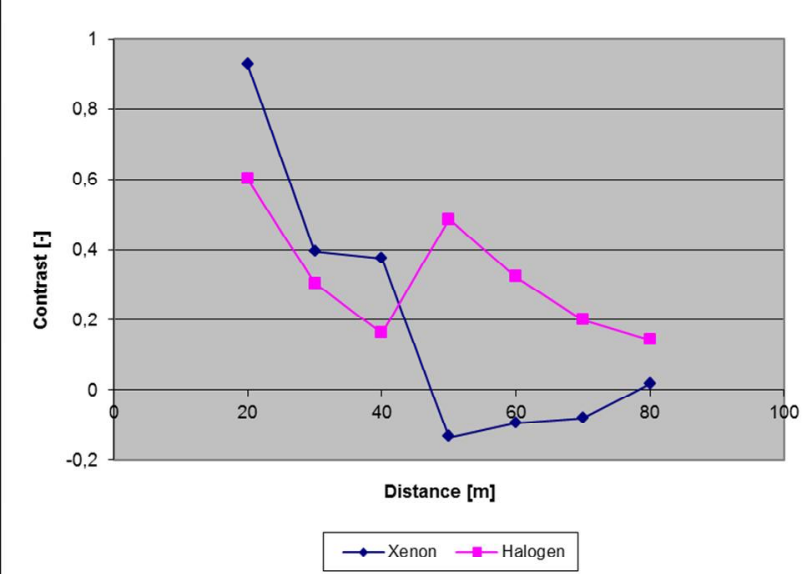

Fig.6. The effect of contrast of a figurant in leisure clothing at certain distances of the vehicle.

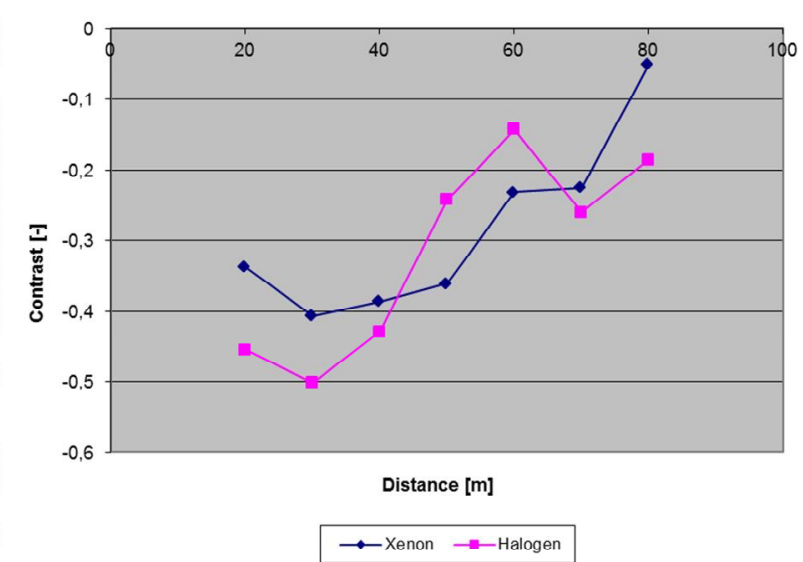

Fig.7. The effect of contrast of a figurant in black clothing at certain distances of the vehicle.

The results indicate clearly, in all cases, that the contrast created by xenon lights is greater up to a distance of about 41 meters, whereupon the contrast created by halogen lights supersedes xenon lights. With black figurants, yet another crossing of these effects can be observed, except that we can afford to ignore this anomaly in view of absolute contrast values. In this context, one may also note the phenomenon of a negative contrast, i.e., situations where the figurant's background has a greater brightness value than the figurant himself.

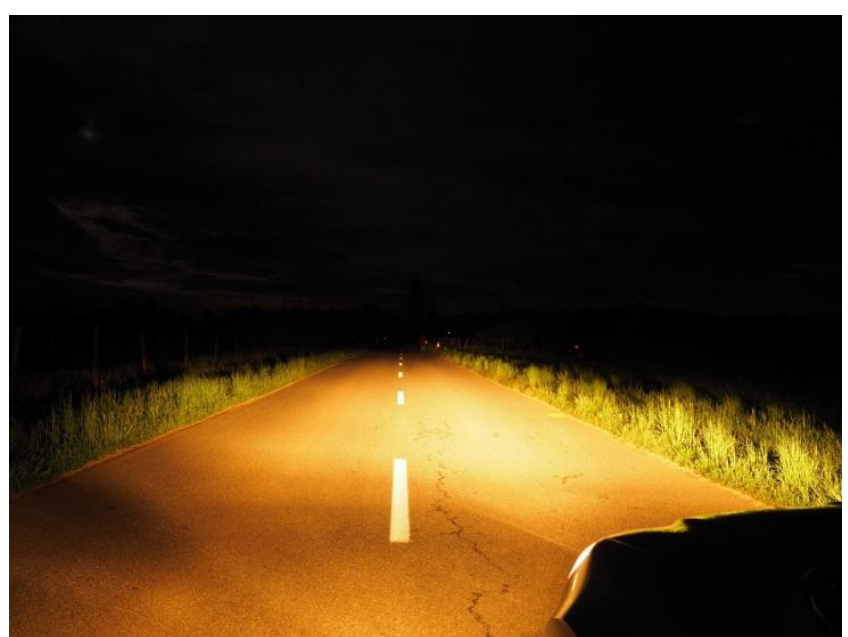

Fig.8. Visibility of a pedestrian in casual (leisure) clothing at a distance of $50 \mathrm{~m}$, including detail - halogen lights

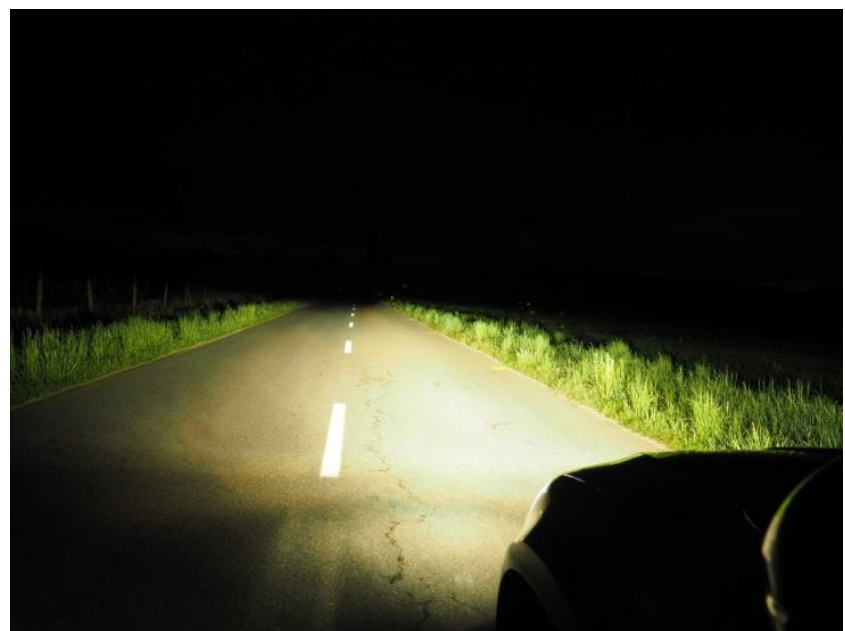

Fig.9. Visibility of a pedestrian in casual (leisure) clothing at a distance of $50 \mathrm{~m}$, including detail - xenon lights

\section{Conclusion}

The measuring results obtained lead to the conclusion that drivers of vehicles fitted with xenon lights are able to see figurants at a shorter distance (i.e., later), than drivers of vehicles with halogen lights. This finding derives from a different dispersion of light in front of the vehicle. While halogen lights have worse illumination parameters and a relatively great dispersion of light to a greater distance from the vehicle, xenon lights illuminate a concrete distance in front of the vehicle up to a sharp line beyond which they practically do not reach at all. In other words, xenon lights illuminate the space up to a certain distance before the vehicle considerably better than halogen lights, but from a distance over approx. 41 meters, i.e., at distances that are critical in rural areas they leave the roadway in the dark (unilluminated), as the sharp line between the illuminated and unilluminated part of the roadway occupies the attention of drivers to the extent where it is harder for them to spot obstructions situated beyond that line. A secondary reason for slower reaction may be the very physiology of the human eye that is capable of accommodating to the light in its surroundings. The strongly illuminated area immediately before a vehicle fitted with xenon lights might cause the iris to shrink, thus making the pupil smaller, so that the eye is unable to perceive the less illuminated area beyond the sharp line of good visibility.

Moreover, it is important to emphasize that our measurements were only taken with a single type of xenon lights, so that the results are not generally applicable. 
Nevertheless, current trends in automotive illumination technology indicate that the development of xenon lights is about to end to make room for LED or laser lights.

\section{Authors:}

Ing. Albert Bradáč, Ph.D., Brno University of Technology, Institute of Forensic Engineering, Purkyňova 464/118, 61200 Brno, e-mail: ing.bradac@usi.vutbr.cz,

Ing. Bc. Marek Semela, Ph.D., Brno University of Technology, Institute of Forensic Engineering, Purkyňova 464/118, 61200 Brno, e-mail:marek.semela@usi.vutbr.cz,

Ing. Jan Skoda, Ph.D., Brno University of Technology, Faculty of Electrical Engineering and Communication, Technická 3082/12, 61600 Brno, e-mail: skoda@feec.vutbr.cz,
Ing. Stanislav Sumec, Ph.D., Brno University of Technology, Faculty of Electrical Engineering and Communication, Technická 3082/12, 61600 Brno, e-mail: skoda@feec.vutbr.cz,

Asoc. prof. Ing. Robert Kledus, Ph.D., Brno University of Technology, Institute of Forensic Engineering, Purkyňova 464/118, 61200 Brno, e-mail: robert.kledus@usi.vutbr.cz.

Ing. et Ing. Martin Bilík, Brno University of Technology, Institute of Forensic Engineering, Purkyňova 464/118, 61200 Brno, e-mail: martin.bilik@usi.vutbr.cz. 